\title{
A CLASS OF GAME SOLUTIONS ${ }^{1}$
}

J. R. ISBELL

1. This note presents a class of finite Neumann-Morgenstern ${ }^{2}$ solutions to certain $n$-person games, corresponding to a transparent principle of organization. If the minimal winning coalitions generally consist of $k$ persons, there may be a finite solution representing the possible formation of any of these ( $k$-person) coalitions, or of certain losing coalitions of $k$ persons. That is, a small losing set may be able to appropriate to itself all the proceeds of the game simply because it blocks the formation of small winning sets (although its large complement wins).

It has been believed, and the author still believes, that a fully developed game theory must take explicit account of the cost of communication and organization. But it is interesting to note that the present theory, without being designed to consider such matters, seemingly does implicitly consider them.

2. The term pseudo-main solution has been in the air for some time, but may be considered to belong to H. M. Gurk, who first proved their existence. ${ }^{3}$ In view of the new possibilities indicated here, we propose the following definition. A pseudo-main solution to a normalized $n$-person game consists of a finite set of imputations $\alpha^{\mathbf{s}}$, associated one-to-one with certain sets $S$ of players; for each player $i$ there is a constant $X_{i}$ such that $\alpha_{i}^{s}=X_{i}$ for $i$ in $S, \alpha_{i}^{s}=0$ for $i$ not in $S$. The set $\left\{\alpha^{s}\right\}$ must form a solution of the game.

Let $\Gamma$ be a game on the set $N$ of $n$ persons such that every $(n-k+1)$-person set contains a $k$-person (completely) winning set. (Hence every set of $k-1$ persons is flat.) Let $A$ be the set of all $k$ person winning sets and $B$ the set of all $k$-person sets not in $A$ whose complements do not contain elements of $A$. Let $V_{k}$ be the set of all the imputations $\alpha^{s}, S$ in $A \cup B$, where $\alpha_{i}^{s}=1 / k$ for $i$ in $S, 0$ otherwise.

(1) Never $\alpha^{s}>\alpha^{T}$. For if $S$ is in $A$, then $T$ meets $S$, and $S-T$ is ineffective; if $S$ is in $B$, then $S$ is ineffective. But $\alpha_{i}^{s}>\alpha_{i}^{T}$ only for $i$ in $S-T$.

(2) Every $\alpha$ not in $V_{k}$ is dominated by some $\alpha^{8}$ in $V_{k}$. For either

(a) $\alpha$ has $k$ coordinates $1 / k$, constituting the set $T$ not in $A \cup B$.

Received by the editors July 30, 1954.

${ }^{1}$ Supported by the Office of Naval Research under Contract N7onr41904.

2 We use D. B. Gillies' normalization. Otherwise terms and concepts not explained here are from von Neumann and Morgenstern, Theory of games and economic behavior.

${ }^{3}$ We do not know Gurk's publication plans. 
Then by definition of $B, N-T$ contains some $S$ in $A$; and $\alpha^{s}>\alpha$, or

(b) $\alpha$ has at least $n-k+1$ coordinates less than $1 / k$, constituting a set $T$ which by hypothesis contains an element $S$ of $A$. Again $\alpha^{s}>\alpha$.

THEOREM. If every $(n-k+1)$-person set contains a $k$-person winning set, then $V_{k}$ is a pseudo-main solution.

3. The class of games just considered includes the direct majority games, where $n=2 k-1$; then $V_{k}$ is the obvious main simple solution. It includes Gurk's first example ${ }^{3}$ of a pseudo-main solution; in this case $n=12, k=4$, and $B$ is empty.

The three-person majority game is the only one having a solution $V_{2}$, as is easily seen. No weighted majority game has a solution $V_{k}$, with the obvious exception of the main simple solution of a direct majority game. For every $k$ of the $2 k-1$ players with largest weights must win.

Of the thirty constant-sum simple games on six or fewer persons, twenty-two have main simple solutions, the others do not, and no more pseudo-main solutions are known. The author knows fifteen pseudo-main solutions of non-constant-sum six-person games, thirteen of which are of the form $V_{3}$.

An example is the simple game with minimal winning sets 123 , $124,156,256,345,346$. Then $V_{3}$ contains fourteen points, corresponding to the six sets listed $(A)$ and the eight sets in $B$. An eight-parameter family of non-simple games, derived from this one by assigning values $V, 0 \leqq V<1$, to the sets in $B$, has the same solution. Of course, if a set in $B$ is made completely winning, then its complement is no longer in $B$ and one point must be removed from the solution.

The author's possibly incomplete list of 671 seven-person constantsum simple games includes 52 with pseudo-main solutions. Twentyone of these are derived from homogeneous weights, and fifteen from the fifteen six-person games mentioned. In the latter cases the seventh player is not useless; he decides ties when the first six players are split into two non-winning three person sets. But in the solution $V_{3}$, the favored six reject his services.

4. We remark in conclusion that every finite projective plane defines a simple game having a pseudo-main solution. If $k$ is the number of elements of the ground field, then the game has $k^{2}+k+1$ players, identified with the points of the plane. The minimal winning sets are the lines. No set of $k$ players meets every line; for each point meets just $k+1$ of the $k^{2}+k+1$ lines. Hence $V_{k+1}$ is a solution. (More care- 
ful counting shows that $B$ is empty.) The seven-person game, $k=2$, is well known.

Added in proof. The 15 solutions mentioned at the end of $\S 3$ are main simple, with $x_{7}=0$. The projective plane games and their solutions $V_{k+1}$ were previously described in an unpublished paper of Moses Richardson.

The George Washington University

\title{
CERTAIN TYPES OF HOMOGENEOUS CONTINUA
}

\author{
C. E. BURGESS
}

According to the usual definition of homogeneity, a point set $M$ is said to be homogeneous if for any two points $x$ and $y$ of $M$ there is a homeomorphism of $M$ onto itself carrying $x$ into $y$. Some more general types of homogeneity previously defined in [2] will be studied in this paper, and it will be shown that there is a certain type of homogeneity such that every decomposable compact metric continuum possessing it is a simple closed curve. For bounded plane continua possessing the usual type of homogeneity, this problem has been only partially solved. ${ }^{1}$ Added in proof. At the Summer Meeting in Laramie, September, 1954, Bing and Jones each presented an example of a decomposable homogeneous bounded plane continuum which is different from a simple closed curve [Bull. Amer. Math. Soc. Abstracts 60-6-766 and 60-6-770].

THEOREM 1. If every proper subcontinuum of the compact metric continuum $M$ is nearly homogeneous, then $M$ is hereditarily indecomposable. $^{2}$

Proof. Since every subcontinuum of $M$ satisfies the hypothesis of this theorem, it will be sufficient to show that $M$ is indecomposable.

Suppose that $M$ is decomposable. Then there is some proper sub-

Presented to the Society, May 1, 1954; received by the editors May 5, 1954 and, in revised form, August 2, 1954. in $[2]$.

${ }^{1}$ For these results, see [3, Theorem 2], [2, Theorem 8], and other references cited [1].

${ }^{2}$ An example satisfying the hypothesis of this theorem has been described by Bing 